säure/Eisen-Supplementation der Anteil von Kindern mit geringem Geburtsgewicht verringert [Wilson RD et al. J Obstet Gyn Can 2015]. In einer Metaanalyse von 41 Studien reduzierte sich ebenso die Zahl der Fehlbildungen bis zu 50\% [Goh et al. J Gyn and Obstet 2013]. Auch Biotin, das „new kid in the block" der Vitaminforscher, sei für die fetale Entwicklung relevant, so Biesalski.

\section{Cave: Mangelernährung bei Senioren}

Einen gesteigerten Bedarf an Mikronährstoffen haben auch Krebspatienten oder adipöse Menschen (Vitamine D, B12, Folsäure sowie $\mathrm{Mg}, \mathrm{Fe}, \mathrm{Cu}, \mathrm{Zn})$, eine verringerte Resorption ist bei $\mathrm{Pa}$ tienten mit Kurzdarm und chronischentzündlichen Darmerkrankungen zu berücksichtigen, erinnerte Biesalski. Eine wichtige Risikogruppe seien außerdem Senioren, bei denen eine Mangelernährung weit verbreitet sei. Defizite bestünden hier vor allem bei den Vitaminen A, B und D. Die Hautsynthese von Vitamin D sei bei Senioren im Vergleich zu jüngeren Menschen deutlich reduziert. Auch Trend-Diäten, die Einnahme sogenannter Fat-Replacer und vegetarische, besonders vegane Ernährung können zu einer Unterversorgung führen, betonte Biesalski und eröffnete damit Prof. Peter Stehle aus Bonn, die Vorlage zu seinem KontraPart. NEM könnten kein Ersatz für eine unausgewogene Ernährung sein, betonte der Ernährungsphysiologe, und ein Nährstoffmangel könne durch geeignete Auswahl von Lebensmitteln behoben oder verhindert werden. Zudem sei noch in keiner Interventionsstudie durch Zufuhr von (hoch dosierten) NEM bei Menschen ohne Mangel an Mikronährstoffen ein präventiver Effekt erzielt worden. Zudem gebe es zu wenige Daten zur langfristigen Sicherheit von NEM. Nicht einmal die optimale Dosierung sei bekannt und wegen großer individueller Unterschiede oft auch schwer zu klären. Es gebe auch nur wenige kritische Nährstoffe. Stehle nannte Folsäure (in allen Altersgruppen) sowie Vitamin D, vor allem bei Kleinkindern und Senioren. Vehement sprach sich der Ernährungsphysiologe gegen die Zufuhr sekundärer Pflanzenstoffe in Kapselform wie Polyphenole, Catechin oder konjugierte Linolsäure aus. Es existierten keine Referenzwerte und der Versorgungsstatus in der Bevölkerung sei nicht zu bewerten, eine
Zufuhr solcher Wirkstoffe also nicht begründbar.

\section{Pro/Contra Nahrungsergän- zungsmittel - Wer gewinnt?}

Ein eindeutiger Gewinner in der Pro und-Kontra-Diskussion war nicht auszumachen, eine Abschlussbewertung durch das Plenum wurde gar nicht erst versucht. So ging die Aufforderung von Dr. Roland Gärtner, Internist aus München, Patienten nach Möglichkeit über NEM aufzuklären, ein bisschen im Mangel guter Evidenzen unter. Dies spricht einerseits für das Fazit von Stehle: Ein bevölkerungsweiter Einsatz von NEM zur Krankheitsvermeidung, Verbesserung des Wohlbefindens und zum Ausgleich diätetischer Missgriffe sei sinnlos. Aber gegen eine gezielte Verwendung von NEM in Risikogruppen ist andererseits sicher kaum etwas einzuwenden.

(RF)

\section{Quelle}

Symposium „Wie sinnvoll sind Nahrungsergänzungsmittel?", 123. Kongress der Deutschen Gesellschaft für Innere Medizin (DGIM), 29.4.2017, Mannheim

\title{
Schützt Schokolade vor Vorhofflimmern?
}

\section{Wer mäßig, aber regelmäßig Schokolade isst, entwickelt möglicherweise seltener ein Vorhofflimmern.}

») Kardiologen haben eine Vorliebe für Schokolade. Das Kakaoprodukt wurde bereits in diversen Indikationen auf mögliche positive Effekte untersucht. Eine Studie aus Dänemark legt jetzt eine inverse Assoziation mit einem symptomatischen Vorhofflimmern nahe.

\section{Prävention zartbitter}

Die 55.502 Teilnehmer der Danish Diet, Cancer, and Health Study waren zu Untersuchungsbeginn im Alter zwischen 50 und 64 Jahren zu ihrem Schokoladenkonsum befragt worden. 3.346 von ihnen entwickelten im Lauf der nächsten 13 Jahre ein symptomatisches Vorhofflimmern. Als Referenzgruppe galten Teilnehmer, die sich seltener als einmal im Monat eine Portion Schokolade (ca. 30 g) gönnten. Im Vergleich dazu war der Konsum von ein bis drei Portionen im Monat mit einem um 10\% geringeren Vorhofflimmernrisiko assoziiert. Bei einer beziehungsweise zwei bis sechs Portionen pro Woche erreichte die Risikoreduktion sogar 17 beziehungsweise 20\%. Wenn die Teilnehmer allerdings Tag für Tag $30 \mathrm{~g}$ und mehr Schokolade aßen, war der Vorteil gegenüber der Vergleichsgruppe nicht mehr signifikant. Bei allen Berechnungen wurde der Einfluss diverser Faktoren wie Geschlecht, BMI, Blutdruck, Cholesterin, Gesamtkalorienzufuhr, Alkoholkonsum und Rauchstatus berücksichtigt.

Eine präventive Wirkung von Schokolade könnte über die Flavanole aus den Kakaobohnen vermittelt werden, vermuten die Autoren. Flavanole sind eine Subgruppe pflanzlicher Polyphenole, die unter anderem gefäßerweiternde, antientzündliche und thrombozytenhemmende Eigenschaften haben.

(bs)

\section{Quelle}

Mostofsky $E$ et al. Chocolate intake and risk of clinically apparent atrial fibrillation: the Danish Diet, Cancer, and Health Study. Heart, online first, 23. Mai 2017 